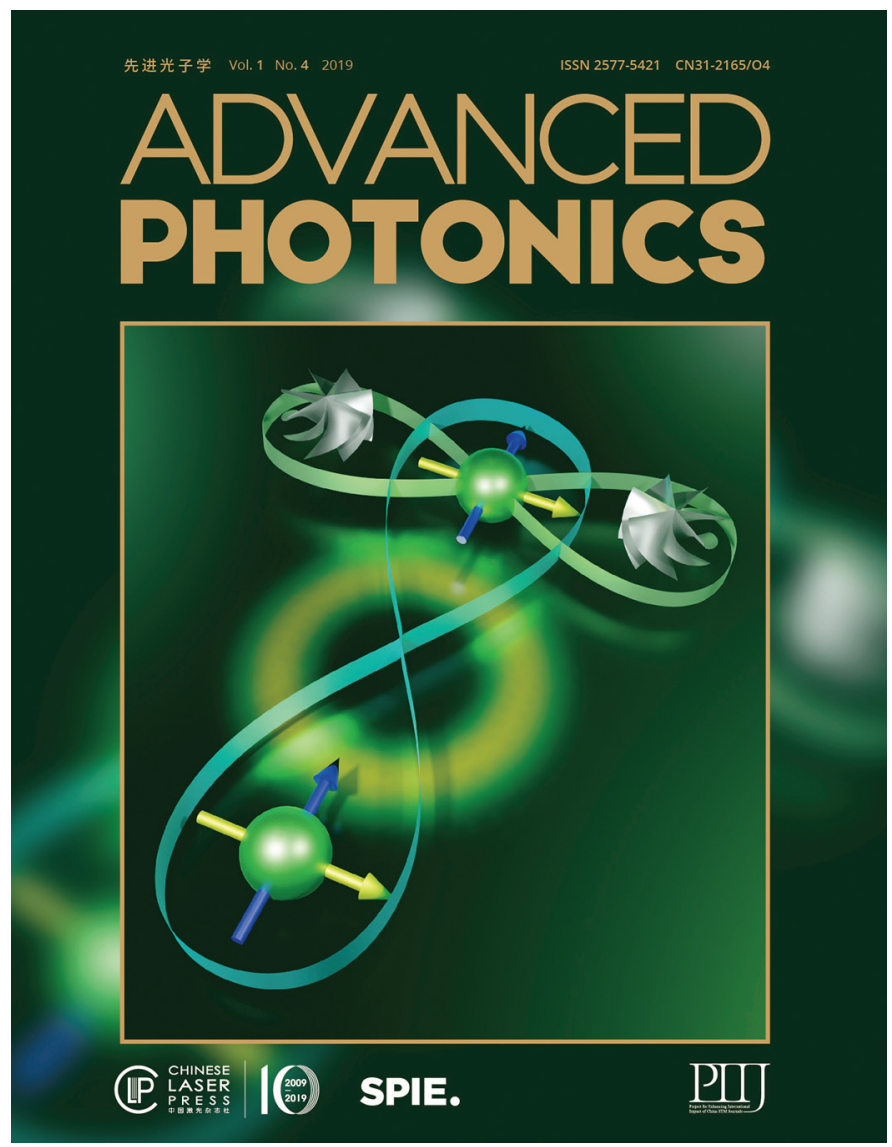

\title{
About the cover: Advanced Photonics Volume 1, Issue 4
}

The image on the cover for Advanced Photonics Volume 1, Issue 4, provides a pictorial view of the hybrid two-photon entangled state. Quantum entanglement is present between the polarization of a first photon (bottom) and the vector vortex state of a second photon (top). The second photon has been transmitted through an air-core fiber and exhibits internal correlations between its orbital angular momentum and its polarization state

The image is based on the research presented by Daniele Cozzolino et al. in their article, "Air-core fiber distribution of hybrid vector vortex-polarization entangled states," Advanced Photonics, 1(4), 046005, doi: 10.1117/1.AP.1.4.046005. 